\title{
Sequencing, Improved Detection, and a Novel Form of Kalanchö̈ top-spotting virus
}

Zihong Yang, Department of Plant Pathology, University of Minnesota, St. Paul 55108; Mogens Nicolaisen, Danish Institute of Agriculture Sciences, Department of Crop Protection, Flakkebjerg, Denmark; Neil E. Olszewski, Department of Plant Biology, University of Minnesota, St. Paul 55108; and B. E. L. Lockhart, Department of Plant Pathology, University of Minnesota

\begin{abstract}
Yang, Z., Nicolaisen, M., Olszewski, N. E., and Lockhart, B. E. L. 2005. Sequencing, improved detection, and a novel form of Kalanchoë top-spotting virus. Plant Dis. 89:298-302.

Virions of Kalanchö̈ top-spotting virus (KTSV) were purified from infected leaf tissue of Kalanchoë blossfeldiana using a procedure that prevented loss of virus in the initial extraction step. The double-stranded DNA viral genome was cloned and sequenced. The KTSV genome was $7,591 \mathrm{bp}$ in size and contained three open reading frames capable of encoding proteins of 21, 14 , and $223 \mathrm{kDa}$, respectively. The size and organization of the KTSV genome were similar to those of other mealybug-transmitted badnaviruses. Several oligonucleotide primer pairs, based on the KTSV genomic sequence, were used to efficiently detect the virus in plants, thereby removing a major constraint to reliable screening of kalanchoë propagating stock and breeding lines for KTSV infection. Two KTSV sequences, one symptom-inducing and the other not, were identified and differentiated by polymerase chain reaction (PCR) amplification and digestion of the resulting amplicon with restriction endonucleases. Preliminary results from graft-transmission tests and PCR indexing suggest that the nonsymptomatic form of KTSV may represent an integrated viral element. The occurrence of such integrated pararetroviral elements poses practical problems for routine PCR indexing of breeding and propagating stock, and also raises the possibility of symptomatic episomal infections arising from these viral integrants.
\end{abstract}

Top-spotting is a disease of kalanchoë (Kalanchoё blossfeldiana) characterized by the appearance of numerous yellow spots on the leaves of affected plants (Fig. $1)$. The disease occurs in many commercially traded cultivars of $K$. blossfeldiana in Europe and North America and can be economically damaging. In 1988, topspotting was shown to be graft, seed, and pollen transmitted and to be associated with the presence of bacilliform virus-like particles (7). These virus-like particles were subsequently shown to be those of Kalanchö̈ top-spotting virus (KTSV), identified as the causal agent of top-spotting (10) and consisting of a doublestranded DNA genome with physicochemical properties similar to those of other badnaviruses (12). Although KTSV

Corresponding author: B. E. L. Lockhart E-mail: lockh002@umn.edu

Current address of Z. Yang: Fred Hutchinson Cancer Research Center, Seattle, WA.

This research was supported in part with funds from the Minnesota Agricultural Experiment Station to B. E. L. Lockhart.

KTSV sequence: GenBank accession no. AY180137.

Accepted for publication 28 October 2004

DOI: 10.1094/PD-89-0298

(C) 2005 The American Phytopathological Society can be transmitted by mechanical inoculation and by the citrus mealybug, Planococcus citri Russo (3), natural spread of top-spotting in commercially grown kalanchoë occurs primarily by vegetative propagation of infected stock plants. Therefore, it is essential to have reliable indexing methods which can be used to identify virus-free propagating stock. In addition, because KTSV is also pollen and seed transmitted in kalanchoë, a similar need for reliable indexing procedures also exists for breeding lines. Until now, however, no method for efficient detection of KTSV infection in kalanchoë has been available. This has been due to a number of factors, including sporadic and unpredictable symptom appearance, difficulty of mechanical transmission and lack of herbaceous indicator plants, and failure to obtain significant amounts of purified virions due to low concentration in and difficulty of extraction from infected tissue $(3,10)$. Because of these constraints, no effective biological or serological indexing procedures for KTSV have existed. Limited success was obtained using degenerate Badnavirus-specific primers (11) for detection of KTSV by polymerase chain reaction (PCR) amplification using total cellular DNA. This procedure, however, does not unambiguously identify KTSV, and does not distinguish between genomic sequences of KTSV and those of other retro elements which may be present in the host plant genome. The purpose of this

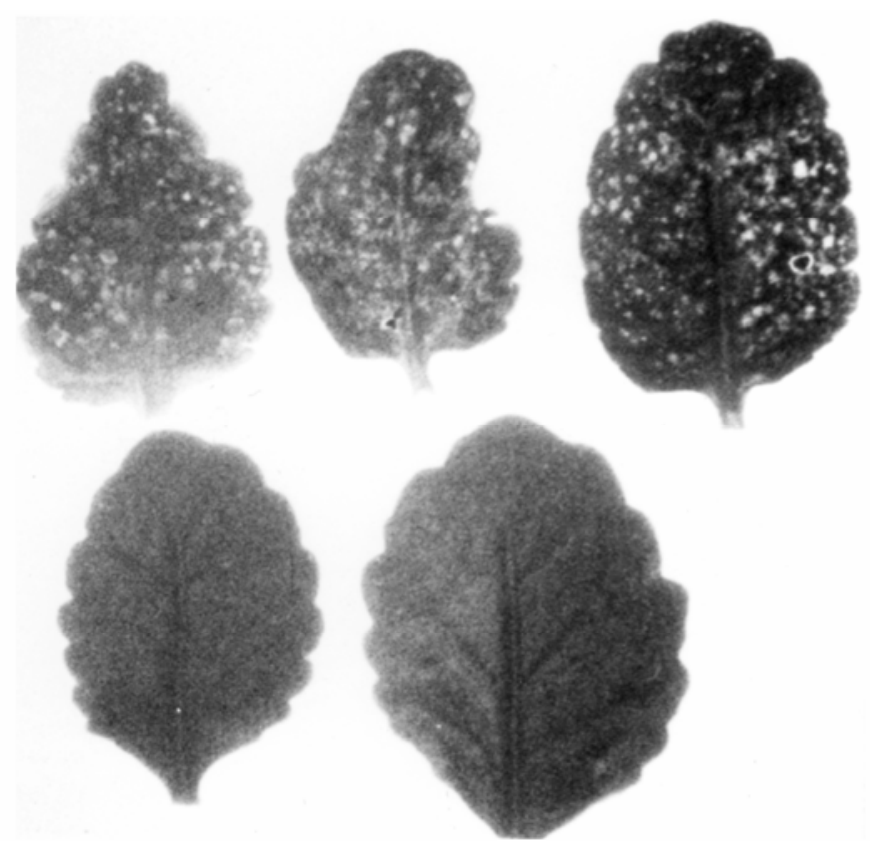

Fig. 1. Symptoms of top-spotting disease in Kalanchoë blossfeldiana cv. Caprice. Diseased (top row) and healthy (bottom row) leaves were placed on a light box and photographed. 
investigation was to obtain additional information on the structure and organization of the KTSV genome, and to develop reliable, efficient genome-based methods for detection of KTSV infection in kalanchoë.

\section{MATERIALS AND METHODS}

Plant and virus sources. Because KTSV occurs frequently in mixed infections with a Carlavus sp., Kalanchoë latent virus (KLV) (6) and a Potyvirus sp., Kalanchö̈ mosaic virus (KMV) (8), a preliminary screening was done by electron microscopic (EM) examination of partially purified leaf extracts (10) to identify $K$. blossfeldiana plants infected only by KTSV. Of nine cultivars showing typical top-spotting symptoms, the cvs. Caprice and Etna were found to contain only KTSV, and Caprice was selected as source plant for virus extraction. The EM-indexed plants were propagated by cuttings and grown under medium shade at 20 to $23^{\circ} \mathrm{C}$, conditions under which symptom expression and virus titer were at their highest (3). K. blossfeldiana cultivars with and without symptoms were obtained from kalanchoë breeders and from Annemarie van Zaayen, Naktuinbouw, Roelofarendsveen, The Netherlands. Virus isolates were maintained in vegetatively propagated $K$. blossfeldiana.

Virus purification and genomic DNA extraction. Initial attempts to purify KTSV from infected kalanchoë leaf tissue resulted in very low yields because virions co-precipitated with host plant material in a coagulum that formed rapidly after tissue maceration $(3,10)$. It was found that coagulation of leaf tissue extracts could be prevented by addition of $6 \%(\mathrm{wt} / \mathrm{vol})$ polyvinylpyrrolidone (PVP; average molecular weight, 40,000) to the extraction buffer. Subsequently, KTSV was purified from infected leaves of the cultivar Caprice showing prominent top-spotting symptoms by extracting fresh or frozen leaf tissue 1:2 (wt/vol) in $200 \mathrm{mM}$ sodium borate, $\mathrm{pH} 8.5$, containing $6 \%(\mathrm{wt} / \mathrm{vol}) \mathrm{PVP}, 0.2 \%$ (wt/vol) $\mathrm{Na}_{2} \mathrm{SO}_{3}$, and $0.1 \%$ (wt/vol) sodium diethyl dithiocarbamate (Na-DIECA). Triton X100 (final concentration $2.5 \% \mathrm{vol} / \mathrm{vol}$ ) was added to the filtered extract, which was stirred for $30 \mathrm{~min}$ at room temperature and then clarified by low-speed centrifugation at $18,000 \times g(\max )$ for $15 \mathrm{~min}$. The clarified supernatant was layered over a 5-ml cushion of $30 \%$ (wt/vol) sucrose in 100 $\mathrm{mM}$ Tris- $\mathrm{HCl}, \mathrm{pH} \mathrm{7.5}$, and centrifuged at $148,000 \times g(\max )$ for $70 \min$ at $12^{\circ} \mathrm{C}$ in a Beckman 50.2 Ti rotor. The resulting pellets were resuspended in $100 \mathrm{mM}$ Tris$\mathrm{HCl}$, pH 7.5, layered on preformed $\mathrm{Cs}_{2} \mathrm{SO}_{4}$-sucrose step gradients (10), and centrifuged at $140,000 \times g(\max )$ for $7 \mathrm{~h}$ in a Beckman SW 28.1 rotor. Gradient columns were fractionated from the bottom using Fluorinert FC-40 (Sigma-Aldrich, St. Louis, MO) as chase liquid. Fractions $(500 \mu \mathrm{l})$ were collected and examined by electron microscopy using negative staining with $2 \%$ (wt/vol) sodium phosphotungstate (PTA), pH 7.0. Gradient fractions containing KTSV virions not contaminated with host plant debris were pooled, diluted 1:4 with $100 \mathrm{mM}$ Tris- $\mathrm{HCl}, \mathrm{pH} \mathrm{7.5,} \mathrm{and}$ centrifuged for $70 \mathrm{~min}$ at $148,000 \times g$ (max). The pelleted virus was resuspended in $10 \mathrm{mM}$ Tris- $\mathrm{HCl}$ and $1.0 \mathrm{mM} \mathrm{MgCl}_{2}$. This constituted the purified KTSV preparation. Genomic DNA was extracted from purified virions that had been treated with DNase and RNase to destroy any residual plant nucleic acids. The encapsidated nucleic acids then were released from the virions by digestion with proteinase $K$ and extracted using phenol-chloroform as described previously (9).

Construction and mapping of genomic clones. After some preliminary restriction mapping of KTSV genomic DNA, a unique ClaI site was used to clone KTSV DNA into the corresponding site of pBluescript $\mathrm{KS}^{-}$(Stratagene, La Jolla, CA) to produce pKTSV19.

DNA sequencing. The sequence of the KTSV insert in pKTSV19 was done by the dideoxy nucleotide chain-termination method using a Sequenase kit (version 2.0; United States Biochemicals, Swampscott, MA) according to the manufacturer's instructions. Comparison between this sequence and those of other badnaviruses suggested that the original KTSV clone (15) lacked a portion of the open reading frame (ORF) I-ORF II region. PCR amplification was used to check this hypothesis. Two oligonucleotides, KTSV-+RNA (5' TGGTATCAGAGCATGGTT 3') and KTSV-
$1000 \quad$ (5' ATGCATAGTTTGGATCCTTA $\left.3^{\prime}\right)$ were designed and used to amplify the region believed to contain the deletion. A $1.8-\mathrm{kb}$ fragment was amplified from KTSV virion DNA, whereas only a $1.0-\mathrm{kb}$ amplicon was obtained from pKTSV19. The 1.8-kb amplicon was gel purified and cloned into pCR2.1 using a TA cloning kit (Invitrogen, Carlsbad, CA) according to the manufacturer's instructions. The KTSV portion of the resulting plasmid was sequenced and the information used to assemble the complete KTSV sequence. Computer analysis of the KTSV DNA sequence and deduced protein sequences encoded by the ORFs were performed using the GCG sequence analysis software package (version 8.0; UNIX, HewlettPackard, Palo Alto, CA) (2).

DNA extraction, PCR amplification, and analysis of PCR products. Total genomic DNA was extracted from $K$. blossfeldiana using the DNeasy Plant Mini Kit (QIAGEN, Hilden, Germany) according to the manufacturer's instructions. The initial homogenization of leaf tissue with a mortar and pestle was done either in liquid nitrogen as recommended by the manufacturer or in $800 \mu \mathrm{l}$ of the supplied homogenization buffer. In the final step, DNA was eluted once with $100 \mu$ l of elution buffer.

Primers for PCR detection were designed on the basis of the nucleotide sequence of the KTSV genome (Table 1). The primer pair KTSV IIIaa forward and KTSV IIIaa reverse, which flank the region defined by the KTSV IIIa primer pair, were used to confirm the sequence of the KTSV IIIa amplicon, including the IIIa primer sites. PCR was performed in a total volume of $25 \mu \mathrm{l}$ containing $10 \mathrm{mM}$ TrisHCI, pH 9.0, $50 \mathrm{mM} \mathrm{KCl,} 1.5 \mathrm{mM} \mathrm{MgCl}_{2}$, $0.1 \%$ Triton X-100, $0.2 \mathrm{mM}$ each dNTP, 1 $\mu \mathrm{M}$ each primer, and 1 unit of Taq DNA polymerase (Promega-Corp., Madison, WI) on a GeneAmp PCR System 9700 (Applied Biosystems, Foster City, CA). The cycling conditions were $95^{\circ} \mathrm{C}$ for 2 min followed by 35 cycles of $15 \mathrm{~s}$ at $95^{\circ} \mathrm{C}$, $30 \mathrm{~s}$ at $64^{\circ} \mathrm{C}$, and $60 \mathrm{~s}$ at $72^{\circ} \mathrm{C}$. At the end of the program, the reactions were held at $72^{\circ} \mathrm{C}$ for $10 \mathrm{~min}$. PCR product $(5 \mu \mathrm{l})$ was restriction digested using two units of MspI or NarI (New England Biolabs, Bev-

Table 1. Primers used in this study

\begin{tabular}{llc}
\hline Primer & \multicolumn{1}{c}{ Sequence $\mathbf{( 5}^{\prime} \mathbf{- 3}^{\prime} \mathbf{)}$} & Position $^{\mathbf{a}}$ \\
\hline KTSV I forward & CTG CTA AGC CAA CCA AAG GAG AT & $897-919$ \\
KTSV I reverse & TGG AGG TAG ATG AGA GTG TTG AGC & $1,147-1,170$ \\
KTSV IIIa forward & TCC CGA GGC CAC CAA CTG ATT TAT & $1,948-1,971$ \\
KTSV IIIa reverse & ACG GGT CCG AAG TTG AGA TGG TTG & $2,263-2,286$ \\
KTSV IIIb forward & TCG GAG TGG CAG CAA GGA TA & $3,425-3,444$ \\
KTSV IIIb reverse & TCA AAG CCG ACG GAG ACT ACA T & $3,788-3,809$ \\
KTSV IIIc forward & TGC GTG CGG AGA ATA GGG ACA T & $3,684-3,705$ \\
KTSV IIIc reverse & CAG CTT GGT TTG CCG ACT AA & $4,129-4,151$ \\
KTSV IIIaa forward & CGA GAT ACC CGA TGG ACA CGA & $1,876-1,896$ \\
KTSV IIIaa reverse & CAA GGG CGG ACT CAG TAT CAT CTC & $2,435-2,458$ \\
\hline
\end{tabular}

${ }^{a}$ Position refers to the position in the Kalanchoë top-spotting virus (KTSV) genome. 
erly, MA) for KTSV IIIa and IIIc amplification products, respectively. Restriction digests were analyzed on a $2 \%$ agarose gel and visualized by ethidium bromide staining. The sequences of the KTSV IIIa and aa amplicons were determined as described above.

Transmission experiments. To determine whether asymptomatic plants that gave a KTSV-positive PCR product contained a transmissible viral entity, and whether such plants could themselves be infected by the KTSV isolate from symptomatic plants, graft transmission experiments were conducted because transmission of KTSV by mechanical inoculation is unreliable (10). In the first experiment, asymptomatic PCR-negative scions were grafted on a total of 18 asymptomatic PCR-positive rootstocks and on 6 symptomatic PCR-positive rootstocks. Second, asymptomatic PCR-positive scions were grafted onto a total of nine symptomatic PCR-positive rootstocks. Plants were kept in the greenhouse for 10 months post grafting and scored for symptom expression in new scion growth by visual observation and for detection of KTSV by PCR using the KTSV IIIa primer pair.

\section{RESULTS}

Virus purification, genomic DNA extraction, cloning, and sequencing. The method described above avoided extensive loss of virus due to co-precipitation with host plant components in the initial extraction step, and yielded virion preparations that were largely free of contamination by plant material (Fig. 2). Genomic DNA extracted from purified virions had an electrophoretic migration pattern in agarose gels similar to that described previously for KTSV and other badnaviruses $(9,12$; data not shown).

Restriction mapping of the KTSV genome indicated that it contained a unique ClaI site. A putatively full-length KTSV clone, pKTSV19, was prepared and sequenced. Analysis of the resulting sequence suggested that an internal portion

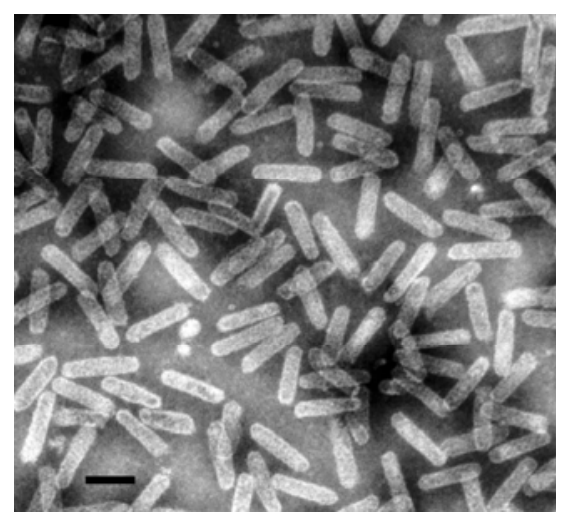

Fig. 2. Virions of Kalanchoë top-spotting virus in a purified preparation. Negative staining with $42 \%$ sodium phosphotungstate, $\mathrm{pH}$ 7.0. Scale bar equals $100 \mathrm{~nm}$. of the genome was deleted in pKTSV19. PCR amplification of the region putatively containing the deletion was used to determine if the missing region was present in virion DNA. This analysis indicated that pKTSV19 carried a 0.8 -kb deletion relative to KTSV DNA from virions (data not shown). The complete DNA sequence for KTSV was assembled from sequences of both genomic clones and the PCR product containing the missing sequence. The results indicated that KTSV has a circular genome of 7,591 bp (GenBank accession no. AY180137).

KTSV contains three large ORFs (Table 2; Fig. 3). The predicted molecular masses of the proteins encoded by these ORFs are similar to those of the corresponding proteins of other badnaviruses $(12,14)$. The genome contains a tRNA ${ }^{\text {Met }}$ binding site that is a hallmark of pararetroviruses. Following the convention established for other badnaviruses, the $5^{\prime}$ end of the tRNA ${ }^{\text {Met }}$ binding site is position 1 on the DNA sequence. The arrangement of the ORFs for these proteins is identical to that of other badnaviruses. Database searches indicate that the KTSV proteins are most similar to the corresponding proteins of other badnaviruses. The similarity and identity be-

Table 2. Comparison of Kalanchoë top-spotting virus and other Badnavirus proteins

\begin{tabular}{lcccc}
\hline & & \multicolumn{3}{c}{ Percent identity (percent similarity) } \\
\cline { 3 - 5 } Protein $^{\mathbf{b}}$ & Size (aa) $^{\mathbf{c}}$ & BSV & ComYMV & ScBV \\
\hline ORF1 & 173 & $37(48)$ & $32(49)$ & $31(43)$ \\
ORF2 & 124 & $36(51)$ & $33(47)$ & $28(43)$ \\
ORF3 & 1,941 & $61(71)$ & $45(57)$ & $42(54)$ \\
\hline
\end{tabular}

a Accession numbers: BSV = Banana streak virus (4): NP_569148, NP_569149, and NP_569150; ComYMV = Commelina yellow mottle virus (13): NP_039818, NP_039819, and NP_039820; and ScBV = Sugar cane bacilliform virus (1): S27936, S27937, S27938.

${ }^{\mathrm{b}} \mathrm{ORF}=$ open reading frame.

c Amino acids (aa).

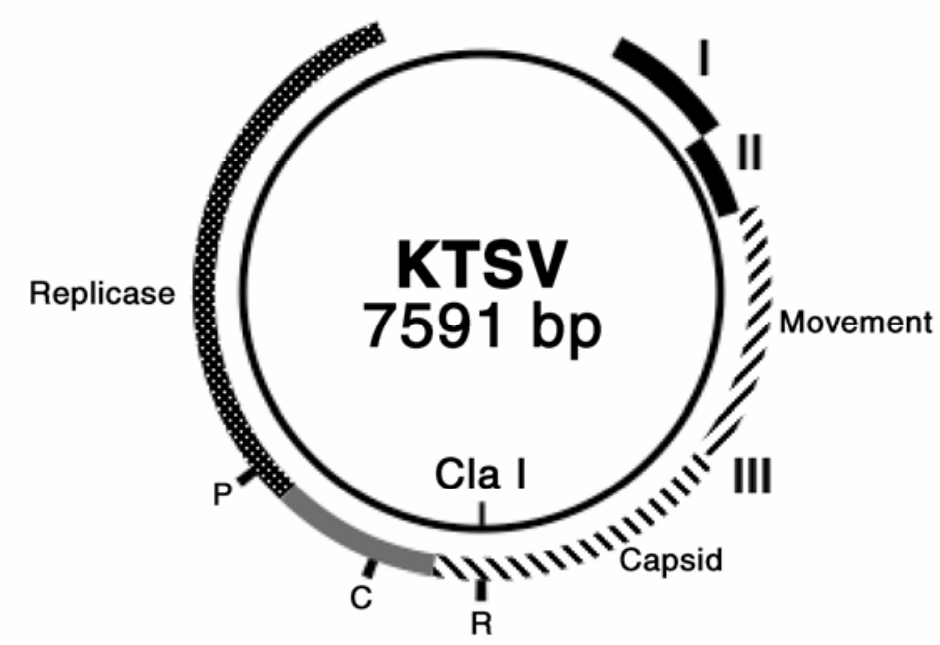

Fig. 3. Map of the Kalanchoë top-spotting virus (KTSV) genome. The arcs outside of the circle indicate the positions of the open reading frames (ORFs). The cell-to-cell movement, capsid, and replicase regions of the polyprotein encoded by ORF III are indicated, as are the RNA binding domain of the capsid region (R), cysteine-rich sequence located between the coat and replicase $(\mathrm{C})$, and active site of the aspartic protease $(\mathrm{P})$ that processes polyprotein. The cloverleaf indicates the location of the

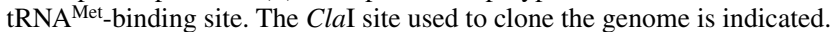


Nucleotide sequence of PCR products from asymptomatic plants. Surprisingly, both primer combinations KTSV IIIa and IIIc gave amplicons from some cultivars with no history of KTSV infection or symptom expression. The nucleotide sequences of the amplicons were determined from three different cultivars and found to be identical between cultivars but different from that of the amplicons obtained from plants with current or previous KTSVinduced symptoms. These nucleotide sequence differences are noted for the KTSV IIIa amplicon in Figure 4. The KTSV IIIa amplicons from symptomatic and asymptomatic plants shared $96 \%$ sequence homology at both the nucleic acid and ORF III protein levels. The four amino acid changes detected in this region were as follows: $\mathrm{N}^{199}-\mathrm{D}, \mathrm{S}^{215}-\mathrm{C}, \mathrm{N}^{251}-\mathrm{D}$, and $\mathrm{G}^{273}$ $\mathrm{S}$. On the basis of this difference in sequence, MspI could distinguish between the two types of amplicons. The KTSV IIIa amplicon from symptomatic plants was digested at position 2,169 , resulting in two fragments of 222 and $116 \mathrm{bp}$, whereas the amplicon from asymptomatic plants was digested at position 1,980, resulting in two fragments of 306 and 32 bp (Fig. 5). Similarly, it was found that an NarI digestion site at position 3,995 was present in the KTSV IIIc amplicon from symptomatic plants but absent from that of asymptomatic plants (data not shown).

Graft transmission experiments. The results of graft transmission experiments are summarized in Table 4. These show that no disease (as assessed by symptom appearance) or KTSV DNA (as assessed by PCR) was transmitted from asymptomatic PCRpositive plants to asymptomatic PCRnegative scions. In contrast, all asymptomatic PCR-positive scions exhibited disease symptoms and contained KTSV DNA following grafting to PCR-positive source plants. Symptoms generally appeared within 1 month after grafting.

\section{DISCUSSION}

Analysis of the KTSV genomic sequence indicates that it contains all features that are diagnostic for badnaviruses (Fig. 3). Thus, KTSV is confirmed as a definitive member of the genus Badnavirus. The plus strand contains the site where tRNA $^{\text {met }}$ binds to prime minus strand synthesis, as well as three large ORFs. Each of the proteins predicted to be encoded by these ORFs is most similar to a protein encoded by members of Badnavirus. The proteins encoded by ORF I, II, and III are most similar to proteins encoded by the corresponding ORFs of Banana streak virus (4; Table 2). The ORF III proteins of badnaviruses are polyproteins consisting of a cell-to-cell movement protein, capsid protein, aspartic protease, reverse transcriptase, and ribonuclease $H(12,14)$. The ORF III protein of KTSV has a similar structure (Fig. 3). The similarity between this protein and other Badnavirus ORF III proteins extends throughout the entire length of the protein. Moreover, the KTSV ORF III protein contains a cysteine-rich sequence $\left(\mathrm{CX}_{2} \mathrm{CX}_{11} \mathrm{CX}_{2} \mathrm{CX}_{4} \mathrm{CX}_{2} \mathrm{C}\right)$ located

between the RNA-binding domain of the capsid and active site of the aspartic protease that processes the polyprotein (Fig. 3). The cysteine-rich sequence is unique to badnaviruses $(1,15)$.

Table 3. Polymerase chain reaction (PCR) detection of Kalanchoë top-spotting virus (KTSV) in 67 Kalanchoë blossfeldiana cultivars and analysis of PCR products by relevant restriction enzymes

\begin{tabular}{lcc}
\hline \multirow{2}{*}{ Analysis } & \multicolumn{2}{c}{ Cultivars in which symptoms have been } \\
\cline { 2 - 3 } & Observed & Never observed \\
\hline Number of cultivars investigated & 22 & 45 \\
Digested by MspI at position 2,169/KTSV IIIa positive & $22 / 22$ & $0 / 26$ \\
Digested by NarI at position 3,995/KTSV IIIc positive & $22 / 22$ & $0 / 26$ \\
KTSV IIIa negative & 0 & 19 \\
KTSV IIIc negative & 0 & 19 \\
\hline
\end{tabular}

1948 TCCCGAGGCCACCAACTGATTTATGTCATCCCAAACATCATCATGACAAT

1998 CAGGGACTTTTACCAGCACATCCAGATGAGCATACTCACTAAAGGGTACA

2048 CAGGATTTCAAGGGGAGGCAAATCTCTTAGTAACTCGAAGCTGTCGATGT

2098 CGACTGACAAGCGTCCCGAATGCTGGGTTCGCTTTCAACATCCAAAGGGT

Fig. 4. Sequence of the amplicon derived from the putative movement domain of open reading frame III using the Kalanchoë top-spotting virus (KTSV) IIIa primer pair. Sequence differences in the polymerase chain reaction products from asymptomatic plants are indicated below the sequence of the KTSV genome, as is the location of the MspI site (double underline) that distinguishes the amplicons obtained from asymptomatic and symptomatic Kalanchoë blossfeldiana plants.

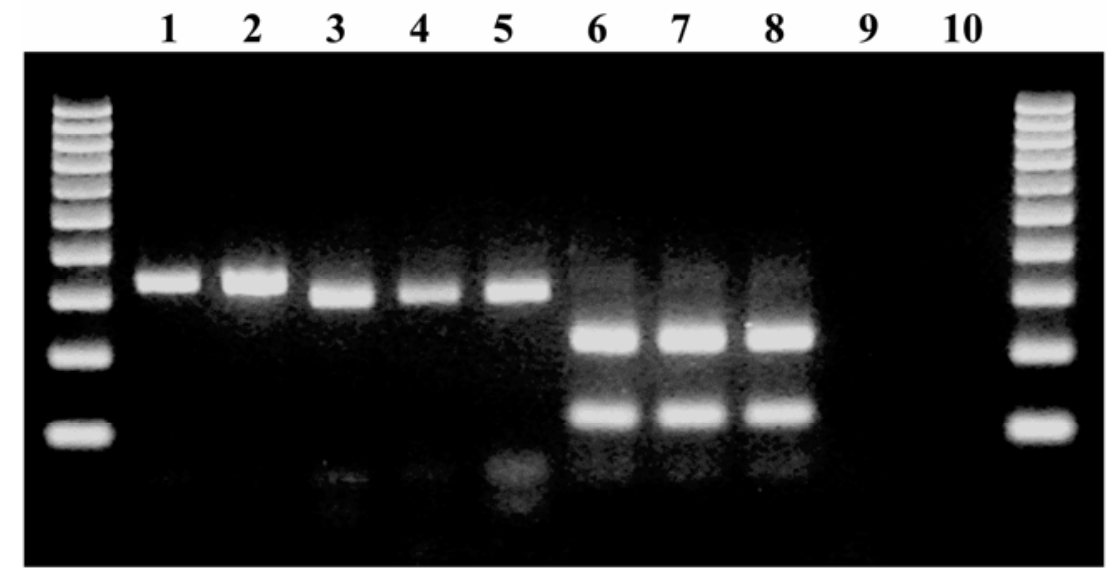

Fig. 5. Agarose gel electrophoretic analysis of polymerase chain reaction (PCR) products obtained from Kalanchoë blossfeldiana DNA using Kalanchoë top-spotting virus IIIa primers and digestion with MspI. The lanes are flanked by a 100-bp DNA ladder (Bio-Rad, Hercules, CA), of which the lowest band is $100 \mathrm{bp}$. The first two lanes are undigested PCR products from an asymptomatic (lane 1) and a symptomatic (lane 2) cultivar. Lanes 3 to 8 are $M s p$ I-digested PCR products from asymptomatic (lanes 3-5) and symptomatic plants (lanes 6-8), respectively. Lanes 9 and 10 represent asymptomatic, PCR-negative plants. 
Table 4. Symptom appearance in and detection of Kalanchö̈ top-spotting virus (KTSV) IIIa polymerase chain reaction (PCR) products from different Kalanchoë blossfeldiana rootstock-scion combinations

\begin{tabular}{lcc}
\hline & \multicolumn{2}{c}{ Scion $^{\text {a }}$} \\
\cline { 2 - 3 } Rootstock & Asymptomatic, PCR-negative & Asymptomatic, PCR-positive \\
\hline Asymptomatic, PCR-positive & $0 / 18$ & $\ldots$ \\
Symptomatic, PCR-positive & $6 / 6$ & $9 / 9$ \\
\hline
\end{tabular}

${ }^{a}$ Of the total number of scions tested, number showing top-spotting and testing positive for KTSV by PCR (number of positive scions/number of scions tested).

Previously, there have been no reliable methods for the rapid and sensitive detection of KTSV. This has been due in part to lack of a reliable immunoassay, primarily because of difficulties in obtaining sufficient quantities of purified virions. This virus is seed and pollen transmissible; therefore, it is of particular interest to kalanchoë breeders to be able to eliminate KTSV-infected plants from current breeding programs or to eliminate the virus from breeding material of potential interest. Because symptom expression and virion titer fluctuate greatly during the year $(3,10)$, more reliable indexing methods are necessary. The present study describes a method for sensitive, reliable detection of KTSV infection in Kalanchö̈ sp. germ plasm. The method consists of a commercial DNA extraction procedure (DNeasy Plant Mini Kit) followed by PCR with the primers KTSV IIIa (forward and reverse), which amplify a 339-bp region of the movement domain of the KTSV ORF III. The method was tested on a wide variety of kalanchoë cultivars and was able to detect KTSV infection in all cultivars in which symptoms had been observed at some point. However, PCR assays and restriction digestion with $M s p I$ led to the identification of a second form of KTSV in kalanchoë cultivars with no history of topspotting disease expression. The former represents an episomal, disease-inducing form of KTSV that is present in symptomatic PCR-positive plants and can be graft transmitted to both asymptomatic PCR-positive and asymptomatic PCR- negative plants. In contrast, the KTSV entity detected in asymptomatic PCR-positive plants was not graft transmitted to asymptomatic PCR-negative plants. This suggests that the latter form of KTSV may represent an integrated pararetroviral element similar to those described for other badna- and caulimoviruses (5). The occurrence in kalanchoë of this putative KTSV integrant poses two potential problems. The first is that, should this integrant be innocuous, routine PCR screening assays will lead to the unnecessary elimination of some breeding lines and commercial propagating stock. The second problem is that the putative KTSV integrant may represent a segment of a potential episomally activatable KTSV sequence similar to Banana streak O'bino L'Ewai virus (BSVOL) in Musa spp., Tobacco vein clearing virus in tobacco, and Petunia vein clearing virus in petunia (5). It is evident that further research is needed to shed light on the nature and biological significance of integrated pararetroviral elements present in kalanchoë and other plant species.

\section{LITERATURE CITED}

1. Bouhida, M., Lockhart, B. E. L., and Olszewski, N. E. 1993. An analysis of the complete sequence of a sugarcane bacilliform virus genome infectious to banana and rice. J. Gen. Virol. 74:15-22.

2. Devereux, J., Haeberli, P., and Smithies, O. 1984. A comprehensive set of sequence analysis programs for the VAX. Nucleic Acids Research 12:387-395.

3. Ferji, Z. 1988. Role of a small bacilliform virus in the etiology of top spotting disease of Kalanchoë blossfeldiana and some properties of the virus. M.S. thesis. University of Minnesota, St. Paul.

4. Harper, G., and Hull, R. 1998. Cloning and sequence analysis of banana streak virus DNA. Virus Genes 17:271-278.

5. Harper, G., Hull, R., Lockhart, B., and Olszewski, N. 2002. Viral sequences integrated into plant genomes. Annu. Rev. Phytopathol. 40:119-136.

6. Hearon, S. S. 1982. A carlavirus from Kalanchoë blossfeldiana. Phytopathology 72:838844.

7. Hearon, S. S., and Locke, J. C. 1984. Graft, pollen, and seed transmission of an agent associated with top spotting in Kalanchoë blossfeldiana. Plant Dis. 68:347-350.

8. Husted, K., Bech, K. Albrechtsen, M., and Borkhardt, B. 1994. Identification, partial sequencing and detection of a potyvirus from Kalanchö̈ blossfeldiana. Phytopathology 84:161-165.

9. Lockhart, B. E. L. 1990. Evidence for a double-stranded circular DNA genome in a second group of plant viruses. Phytopathology 80:127-131.

10. Lockhart, B. E. L., and Ferji, Z. 1988. Purification and mechanical transmission of Kalanchoë top spotting-associated virus. Acta Hortic. $234: 72-78$

11. Lockhart, B. E. L., and Olszewski, N. E. 1993. Serological and genomic heterogeneity of banana streak badnaviruses: implications for virus detection in Musa germplasm. Pages 105-113 in: Breeding Banana and Plantain for Resistance to Diseases and Pests. J. Ganry, ed. CIRAD/INIBAP, Montpellier, France.

12. Lockhart, B. E. L., Olszewski, N. E., and Hull, R. 1995. Genus badnavirus. Pages 185-188 in: Virus Taxonomy: Sixth Report of the International Committee on Taxonomy of Viruses. F. A. Murphy, ed. Springer-Verlag, Vienna, Germany.

13. Medberry, S. L., Lockhart, B. E. L., and Olszewski, N. E. 1990. Properties of Commelina yellow mottle virus's complete DNA sequence, genomic discontinuities and transcript suggest that it is a pararetrovirus. Nucleic Acids Res. 18:5505-5513.

14. van Regenmortel, M. H. V., Fauquet, C. M., Bishop, D. H. L., Carstens, E. B., and Estes, M. K., eds. 2000 Virus Taxonomy: Classification and Nomenclature of Viruses. Rep. Int Comm. Taxon. Viruses, 7th. Academic Press, San Diego.

15. Yang, Z. 1995. An analysis of the nucleotide sequence and genomic organization of Kalanchoë top spotting virus. M.S. thesis. University of Minnesota, St. Paul. 